Research Paper

\title{
Histology, Glycosaminoglycan Level and Cartilage Stiffness in Monoiodoacetate-Induced Osteoarthritis: Comparative Analysis with Anterior Cruciate Ligament Transection in Rat Model and Human Osteoarthritis
}

\author{
Sangeetha Vasudevaraj Naveen ${ }^{1}$, Raja Elina Ahmad ${ }^{\circledR}$, Wong Jia Hui ${ }^{1}$, Abdulrazzaq Mahmod Suhaeb1, \\ Malliga Raman Murali, Rukmanikanthan Shanmugam ${ }^{1}$ and Tunku Kamarul1,2 \\ 1. Tissue Engineering Group (TEG), National Orthopaedic Centre of Excellence in Research and Learning (NOCERAL), Department of \\ Orthopaedic Surgery, Faculty of Medicine, University of Malaya, 50603 Lembah Pantai, Kuala Lumpur, Malaysia. \\ 2. Clinical Investigative Centre (CIC), University Malaya Medical Centre, Kuala Lumpur, Malaysia \\ 3. Department of Physiology, Faculty of Medicine, University of Malaya, 50603 Lembah Pantai, Kuala Lumpur, Malaysia.
}

$\triangle$ Corresponding author: Raja Elina Ahmad, PhD, Department of Physiology, Faculty of Medicine, University of Malaya, 50603, Lembah Pantai, Kuala Lumpur, Malaysia. Tel: +603 79674922, Fax: +603 79674775; E-mail: elina@ummc.edu.my

() Ivyspring International Publisher. This is an open-access article distributed under the terms of the Creative Commons License (http://creativecommons.org/ licenses/by-nc-nd/3.0/). Reproduction is permitted for personal, noncommercial use, provided that the article is in whole, unmodified, and properly cited.

Received: 2013.06.21; Accepted: 2013.12.15; Published: 2013.12.21

\begin{abstract}
Monosodium -iodoacetate (MIA)-induced animal model of osteoarthritis (OA) is under-utilised despite having many inherent advantages. At present, there is lack of studies that directly compare the degenerative changes induced by MIA with the surgical osteoarthritis induction method and human osteoarthritis, which would further verify a greater use of this model. Therefore, we compared the histological, biochemical and biomechanical characteristics in rat model using MIA against the anterior cruciate ligament transection (ACLT) and human cartilage with clinically established osteoarthritis. The right knees of Sprague-Dawley rats were subjected to either MIA or ACLT ( $n=18$ in each group). Six rats were used as controls. Human cartilage samples were collected and compared from patients clinically diagnosed with $(n=7)$ and without osteoarthritis $(n=3)$. Histological, biochemical (Glycosaminoglycans/total protein) and biomechanical (cartilage stiffness) evaluations were performed at the end of the $1^{\text {st }}$ and $2^{\text {nd }}$ week after OA induction. For human samples, evaluations were performed at the time of sampling. Histopathological changes in the MIA group were comparable to that observed in the ACLT group and human OA. The Mankin

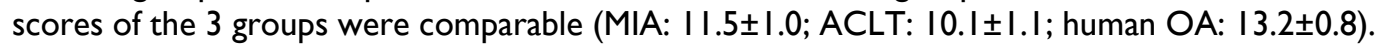
Comparable reduction in Glycosaminoglycan/total protein content in the intervention groups were observed (MIA: $7 \pm 0.6 ; A C L T: 6.6 \pm 0.5$; human OA: $3.1 \pm 0.7$ ). Cartilage stiffness score were $24.2 \pm 15.3 \mathrm{Mpa}$ for MIA, $25.3 \pm 4.8$ for ACLT and $0.5 \pm 0.0 \mathrm{Mpa}$ for human OA. The MIA model produces comparable degenerative changes to $A C L T$ and human $O A$ with the advantage of being rapid, minimally invasive and reproducible. Therefore, wider utilisation of MIA as animal translational OA model should perhaps be advocated.
\end{abstract}

Key words: Animal model; joint; glycosaminoglycan; Mankin score; indentation test; stiffness; monosodium-iodoacetate.

\section{INTRODUCTION}

Osteoarthritis (OA), the clinical syndrome of joint pain and dysfunction, is a recognised major cause of disability, particularly in the aging population (1). The increasing prevalence and absence of cure for the disease has resulted in OA being a substantial health economic burden for the nations. Thus, there is a pressing socioeconomic need for extensive improvements in the prevention and treatment of OA. 
This has led to considerable research efforts being directed in the recent years to improve the understanding of this disease process. Whilst it would be more appropriate to study the pathogenesis of OA in human subjects, such pre-clinical studies may not have been widely conducted owing to a number of challenges. These include difficulty in obtaining sequential human joint tissues throughout the disease progression that is typically slow and variable, and, there is a lack of universally accepted tissue diagnostic criteria or validated surrogate markers to monitor the disease progression and efficacy of treatment $(2,3)$. In addition, human subjects are exposed to a multitude of genetic and environmental factors that may influence the pathology of OA. These may act as confounding factors that could produce inconclusive evidence on the actual clinical efficacy of a particular therapy for OA. In light of this, the use of animal models particularly rats remained valuable and is a more practical approach to study OA. Various methods for inducing OA in rats exist at present (4). Among them, the anterior cruciate ligament transaction (ACLT) model has been widely described and showed the appropriate histological and biochemical changes associated with OA progression (5). ACLT results in joint instability, and thus induces cartilage degeneration, subchondral bone sclerosis and osteophyte formation, which mimics the pathological changes observed in human OA $(6,7)$. Another commonly described OA induction method is the intra-articular injection of monosodium-iodoacetate (MIA). This substance inhibits glyceraldehye-3-phosphate dehydrogenase activity in chondrocytes and thus results in cell death following the disruption of its cellular glycolysis process (8). The progressive loss of chondrocytes leads to changes in the histological and morphological features of articular cartilage, ultimately resulting in changes that closely resemble conditions seen in human OA (9-13). Despite being a convenient model (rapidity, ease of application and consistency in the induction of cartilage damage), whether or not the MIA animal model adequately represent human OA remains debatable. To date, it is still unclear whether the tissue characteristics of the animal subjected to MIA injection are comparable to that of ACLT and human OA, since parallel comparisons between the three conditions have not yet been conducted. This study therefore aims to compare the cartilage tissue characteristics from the knee joint of Sprague-Dawley rats following chemical induction of OA (MIA) against the surgical method (i.e. ACLT) from a more comprehensive perspective, which include the histological, biochemical (GAG expression) and biomechanical (cartilage stiffness) evaluation. In addition, to determine whether these models showed high similarities to human OA, the tissue characteristics in both animal models were compared to those of the human knee joints with clinically established OA.

\section{MATERIAL AND METHODS}

\section{Animals}

Forty-two (N=42), 12-week old Sprague-Dawley (SD) rats weighing between 100 and $150 \mathrm{~g}$, obtained from the Experimental Animal Centre of University Putra Malaysia, were used in this study. All animal experiments were conducted according to the guidelines for animal handling and welfare set by University of Malaya. Approval to conduct this study was granted by the University of Malaya ethics research review committee (OS/05/08/2009/WJH(R)). The rats were divided into three groups: Group $1(\mathrm{n}=18)$ underwent ACLT procedure; group $2(\mathrm{n}=18)$ received intra-articular injection of MIA; and group 3 $(n=6)$ were not subjected to any intervention and were used as controls. OA was induced by these methods only in the right knees of the animals. Within each intervention group, the rats were further grouped into three small subgroups consisting of three animals per subgroup $(\mathrm{n}=3)$ for each of the 3 different analyses (histology, biochemical and biomechanical analysis). The animals were sacrificed using a lethal dose of intraperitoneal ketamine on the $1^{\text {st }}$ and $2^{\text {nd }}$ week after the ACLT procedure and MIA injection to observe the progression of $\mathrm{OA}$ in the animals. Thus, nine rats $(n=9)$ per intervention group were sacrificed at each time point for data comparison. All 6 rats in the control group were sacrificed immediately, since it is expected that in the normal knees, there would not be any apparent change in the cartilage within the short time frame of testing. The normal knee samples harvested for the laboratory analyses represent the control data for both week 1 and 2. In all animals, the whole knee cartilage was resected and subjected to histological, biochemical and biomechanical examinations.

\section{Human tissue samples}

Acquisition of tissues samples from patients was approved by the University of Malaya Medical Centre Ethics committee (Ethics ref. No. 832.12). Knee specimens from humans were collected from 7 patients diagnosed clinically with OA who underwent total knee replacement, and 3 patients without OA who underwent knee surgery e.g. ACL reconstruction or trauma (normal tissue). Clinical diagnosis of OA was done based on the clinical history and examination performed by a senior orthopaedic surgeon. This was further confirmed by the gross morphological changes of the tissues seen during surgery, which include 
very thin cartilage, exposed bone, visible osteophytes and change in cartilage colour from the shiny white usually seen in normal cartilage to a yellowish coloured tissue. In addition, OA in these patients were also diagnosed based on radiological criteria, which include narrow joint spaces, sub-chondrosis and osteophytes formation. As for the group representing the normal cartilage, the patients $(n=3)$ have never been clinically diagnosed with OA nor had previous history of any joint diseases. The absence of OA in this group was further confirmed by radiological investigation, and furthermore, the cartilage appeared normal and deemed to be intact during the operation. The cartilage was taken from young patients and from non-weight bearing sites, which would not be expected to have undergone any OA changes. These patients were trauma patients who were otherwise healthy and had uneventful past medical history. Written informed consent was obtained from each patient prior to the surgery.

\section{Surgical induction of OA by anterior cruciate ligament transection}

Animals were anesthetized using Ketamine hydrochloride $(50 \mathrm{mg} / \mathrm{ml}$ for injection USP, Rotexmedica, Germany) and Xylazine hydrochloride (Ilium Xylazil-20 20mg/ml for injection, Troy laboratories Pty Ltd.). The dose of the anaesthetic agents was measured and delivered according to the body weight of the rats. After sterilisation of the animal's body area, a para-median skin incision was made via the medial para-patellar approach and the patella was retracted laterally. Using micro-surgical instruments, the anterior cruciate ligament-tendon joint was identified, transected and was subsequently washed with normal saline. The surgical wound was then closed in layers.

\section{Chemical induction of OA using Monosodium-iodoacetate injection}

The rats were anesthetized using Ketamine hydrochloride $(50 \mathrm{mg} / \mathrm{ml}$ for injection, USP, Rotexmedica, Germany) and Xylazine hydrochloride (Ilium Xylazil-20 20mg/ml for injection, Troy laboratories PTY limited) and placed in the supine position. The intra-patellar tendon was identified and a single intra-articular injection of $2 \mathrm{mg}$ of Monosodium-iodoacetate (MIA) (Crystal Powder M=185.96 $\mathrm{g} / \mathrm{mol}$, Germany, Sigma) in a total volume of $25 \mu \mathrm{l}$ was injected into the right knee.

\section{Histological evaluation}

Animals were euthanized and the knee joints were dissected to obtain the distal femur. Samples were decalcified in $10 \%$ formic acid for 11 to 14 days. The distal femur was cut with an aim to obtain the center of the medial condyle (i.e. the mechanical weight bearing area) for histology. Samples were dehydrated in ethanol series immersion and embedded in paraffin. For each block, a minimum of ten slides with at least three samples of tissue per slide was obtained to minimise sampling errors. Prior to staining, the paraffin was removed by immersing the slides for two minutes in xylene and ethanol solutions. The slides were divided equally for haematoxylin-eosin (cellular architecture) and Safranin-O-fast green staining (proteoglycan contents of matrix). Histological assessment based on the Mankin scoring system was carried out by two independent observers who were blinded to the study groups. Using this well-established method, the tissues were given scores ranging from $0-14$, with 0 corresponding to a normal joint, and 14 for maximally degenerated joint (14).

\section{Biochemical analysis}

Protein and glycosaminoglycan (GAGs) levels were determined using Bio-Rad DC protein assay kit (Bio-Rad Laboratories; USA) and Blyscan sulfated Glycosaminoglycan assay kit (Biocolor Ltd., UK) according to the manufacturer's protocols. The levels of GAG and total protein were measured in the dissected whole section of the knee i.e. the total surface of the femur and tibia that has not undergone any processing procedure. Spectrophotometric absorbance measurement for protein was obtained at $750 \mathrm{~nm}$ wavelength, while that of GAG was obtained at 656 $\mathrm{nm}$ wavelength. GAG content was normalized to the protein contents and expressed in the unit of $\mu \mathrm{g}$ GAG/mg protein.

\section{Biomechanical evaluation}

Biomechanical testing was performed at week 2 post-intervention. The cartilage stiffness was evaluated by microindentation test using an Instron microtester 5848 attached with a $50 \mathrm{~N}$ load cell. The resolution of testing was $0.01 \mathrm{~N}$ load and $0.01 \mathrm{~mm}$ displacement. The test was performed on the medial femoral condyle and was standardized to a localized area. The harvested femur (fresh specimen) was secured onto a custom made jig such that the area of interest was positioned orthogonal to the indenter. The cartilage was kept hydrated using phosphate buffered saline (PBS). A flat tip conical indenter with a tip diameter of $256 \mu \mathrm{m}$ was placed in contact with the cartilage that was preloaded to $0.01 \mathrm{~N}$. The preload was maintained for 10 minutes until equilibrium was achieved. Ramp load of $0.04 \mathrm{~N}$ at a speed of 10 $\mathrm{mm} / \mathrm{min}$ was used. The initial linear portion of the load displacement curve was used to calculate the stiffness of the cartilage. The biomechanical test was performed in 3 replicates covering 6 different areas, 
and the average value of the data was subsequently used in the statistical analysis.

\section{Statistical analysis}

Results were expressed as mean \pm standard deviation for each parameter examined. The data were analysed using non-parametric test (Mann-Whitney $\mathrm{U})$. Correlations between the histological, biochemical and biomechanical parameters were determined by Spearman's correlation and linear regression analyses. Correlation values were interpreted according to the guidelines of Landis and Koch: 0.00 as poor, $0.00-0.20$ as slight, $0.21-0.40$ as fair, $0.41-0.60$ as moderate, $0.61-0.80$ as substantial, and $0.81-1.00$ as almost perfect (15). A $p$ value of $<0.05$ was considered statistically significant. All analyses were conducted using the statistical software SPSS version 17.0.

\section{RESULTS}

\section{Histology}

Cartilage degeneration and abrasion were evident in all OA specimens originating from both animal and human tissues. In the rats, cartilage tissues from the normal knee (from the control group) showed normal histological appearance and proteoglycan content (Fig. 1A), whereas those subjected to ACLT and MIA injection showed varying degrees of joint degeneration at week 2 post-intervention, which were comparable to each other (Fig. 1B and 1C).

The Mankin scores throughout the duration of the experiment are summarised in figure 2 . The control group has the lowest score (0), reflecting good cartilage tissue quality. The expected characteristics of a normal cartilage tissue including a preserved structural integrity of the continuous articular surface, intact tidemark, normal cellular distribution and orientation, and normal staining with Safranin-O were observed in this group. In contrast, the MIA group had a mean score of $6.0 \pm 0.9$ in the $1^{\text {st }}$ week and $11.5 \pm 1.0$ at the end of week 2 (Fig. 2A), and the histopathological changes were similar to that observed in the ACLT group (ACLT at week $1=7.2 \pm 1.2$ and week $2=10.1 \pm 1.1$ ). The Mankin score for both intervention groups at week 2 post-intervention appeared to be comparable to that of the human OA condition $(13.2 \pm 0.8)(\mathrm{p}>0.05)$ (Fig.2B).

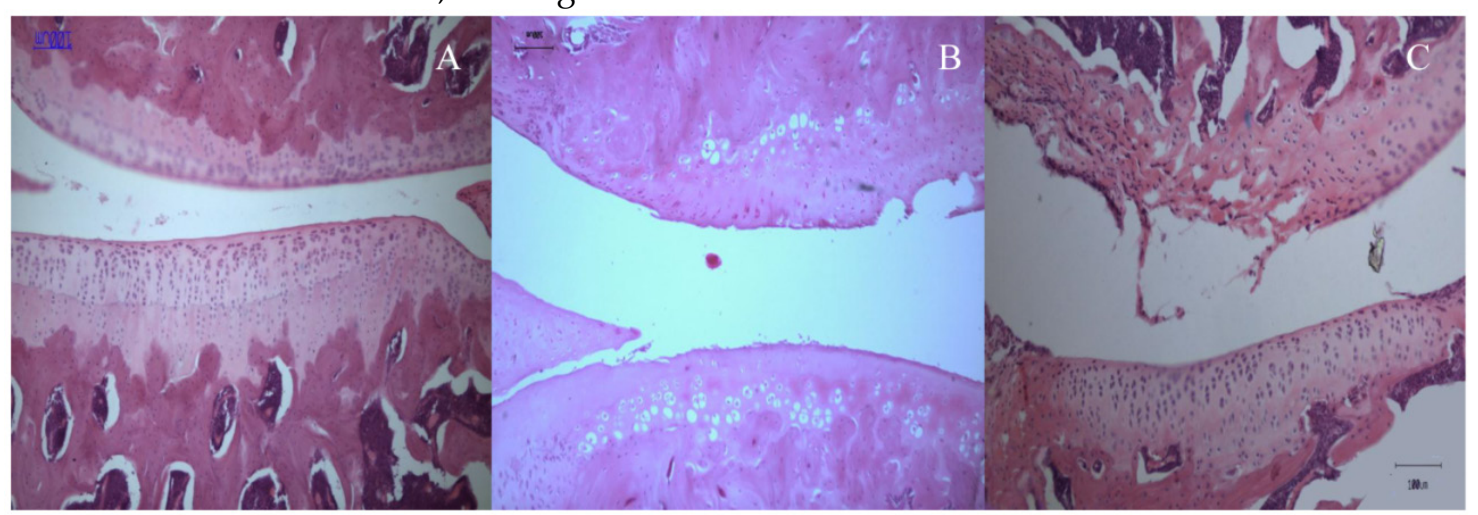

Figure I: Sagittal section through the medial femoral condyles of rat (IOX).(A) Normal articular cartilage structures showing smooth articular surface, normal chondrocytes with columnar orientation, intact tide mark and subchondral bone (Mankin score - 0). (B) At 2 weeks post-ACLT, a confined area of cartilage destruction was observed as reflected by the irregularity of the articular surface and moderately reduced chondrocyte numbers. The tidemark remained visible and the subcondral bone remained intact (Mankin score - 10). (C) At 2 weeks post-MIA injection, more destructive changes over the femoral condyle was observed, with the loss of articular cartilage integrity, moderate to severe reduction in chondrocytes that appear to be necrotic and largely dispersed. The tide mark was not clearly visible (Mankin score - II).
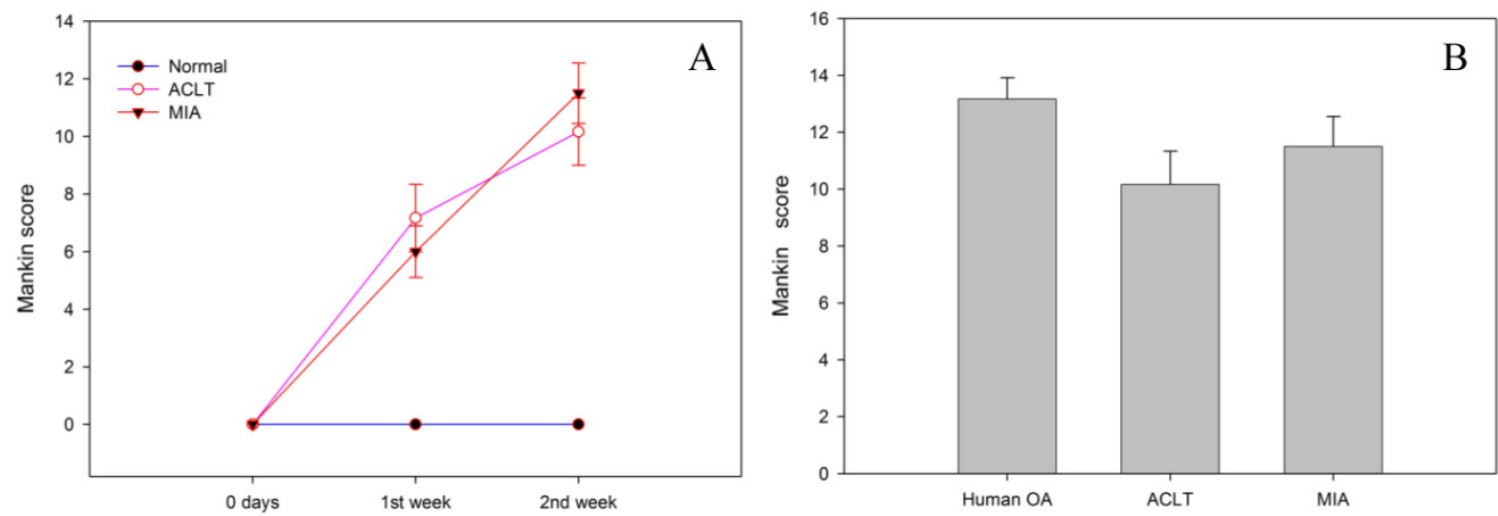

Figure 2: A) The use of MIA causes increased cartilage degeneration as reflected by the increase in Mankin scores overtime, which is comparable to that of ACLT $(p>0.05)$. B) The histological scores for both animal models of OA at week 2 post-intervention were comparable to that of human $O A$ ( $P>0.05)$. 


\section{Biochemical Analysis}

Fig $3 \mathrm{~A}$ and $3 \mathrm{~B}$ represents the GAG/total protein of tibia and femur for MIA and ACLT groups, respectively. The results show that the GAG/total protein was significantly lower in tibia and femur in both intervention groups compared to the control group $(p<0.05)$; however, in the intervention groups, the GAG/protein content was significantly lower in the femur than in tibia at both time points $(\mathrm{p}<0.05)$ (table 1 and 2). No significant difference was observed between week 1 and week 2 in the ACLT group, which indicates that ACLT has reached its maximal damaging effect as early as week 1 and this persisted till week 2. However, in the MIA group, the GAG/total protein content was significantly lower in week 2 compared to week1, indicating that considerable damage to the cartilage may have occurred at a relatively slower pace when $\mathrm{OA}$ is induced by this method. However, no significant difference was observed between the MIA and ACLT group at week 2, indicating similar extent of damage in both groups at the end of week 2. Nevertheless, the animal tissues in both intervention groups had significantly higher GAG/total protein level than that in the human OA tissue samples. In the human OA samples, the GAG/total protein level was significantly lower in the
OA samples $(3.1 \pm 0.7)$ as compared to the normal knees (13.5 \pm 2.5$)$, indicating a relatively lower extracellular matrix components in the degenerated cartilage tissues $(\mathrm{P}<0.05)$ (Fig.3C).

Table I: GAG/total protein concentration in Tibia

\begin{tabular}{lllll}
\hline Week & Group & $\begin{array}{l}\text { Mean } \pm \text { Standard } \\
\text { Deviation }\end{array}$ & \multicolumn{2}{c}{$\begin{array}{l}\text { Comparison between } \\
\text { groups (P values) }\end{array}$} \\
\cline { 3 - 5 } & & & $0.002^{*}$ & NIL \\
\hline Week 1 & ACLT & $14.6 \pm 1.3$ & $0.02^{*}$ & $0.02^{*}$ \\
& MIA & $16.8 \pm 1.5$ & NIL & $0.002^{*}$ \\
& Normal & $25.6 \pm 4.8$ & $0.002^{*}$ & NIL \\
Week 2 & ACLT & $12.9 \pm 1.1$ & $0.002^{*}$ & $0.02^{*}$ \\
& MIA & $10.9 \pm 0.9$ & NIL & $0.002^{*}$ \\
& Normal & $25.6 \pm 4.8$ & &
\end{tabular}

*-Significant, $\mathrm{P}<0.05$.

Table 2: GAG/total protein concentration in Femur

\begin{tabular}{lllll}
\hline Week & Group & $\begin{array}{l}\text { Mean } \pm \text { Standard } \\
\text { Deviation }\end{array}$ & $\begin{array}{l}\text { Comparison between } \\
\text { groups (P values) }\end{array}$ \\
\cline { 3 - 5 } Wormal & ACLT \\
\hline Week 1 & ACLT & $6.8 \pm 0.6$ & $0.002^{*}$ & NIL \\
& MIA & $12.1 \pm 1.1$ & $0.002^{*}$ & $0.002^{*}$ \\
& Normal & $22.9 \pm 4.6$ & NIL & $0.002^{*}$ \\
Week 2 & ACLT & $6.6 \pm 0.5$ & $0.002^{*}$ & NIL \\
& MIA & $7.1 \pm 0.6$ & $0.002^{*}$ & 0.394 \\
& Normal & $22.9 \pm 4.6$ & NIL & $0.002^{*}$ \\
\hline
\end{tabular}

*-Significant, $\mathrm{P}<0.05$
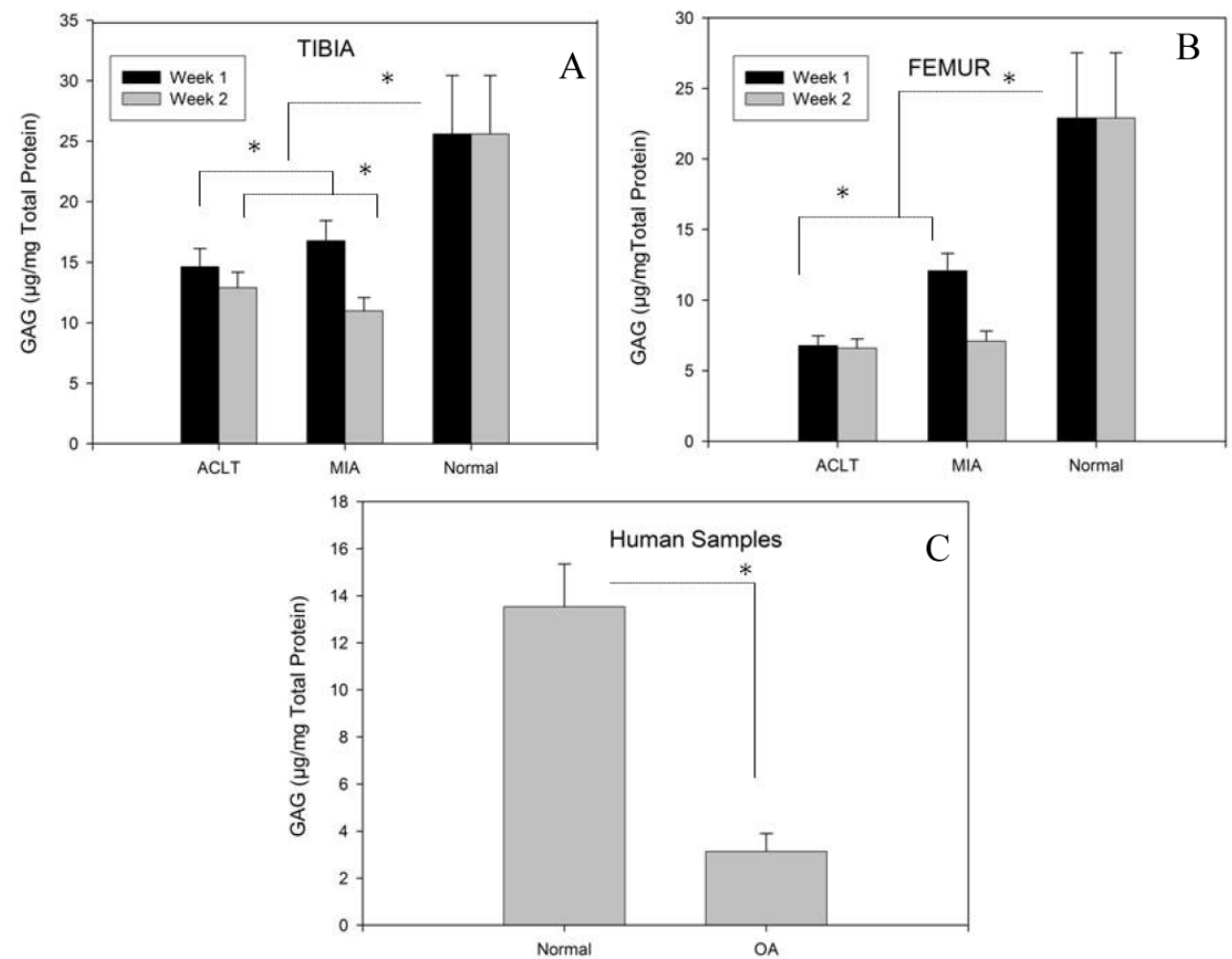

Figure 3: Compared to the normal tissues, GAG/total protein levels were lower in both intervention groups at the tibia (A) and femur (B). Although at week I, the GAG/total protein levels were higher in the MIA group compared to ACLT at both tibia and femur, by week 2, the concentrations had dropped to a level comparable to that in the ACLT group, specifically in femur. In human samples, significant reduction of GAG/total protein was observed in the OA samples compared to normal tissue $(C)(*$ indicates significance $p<0.05)$. 

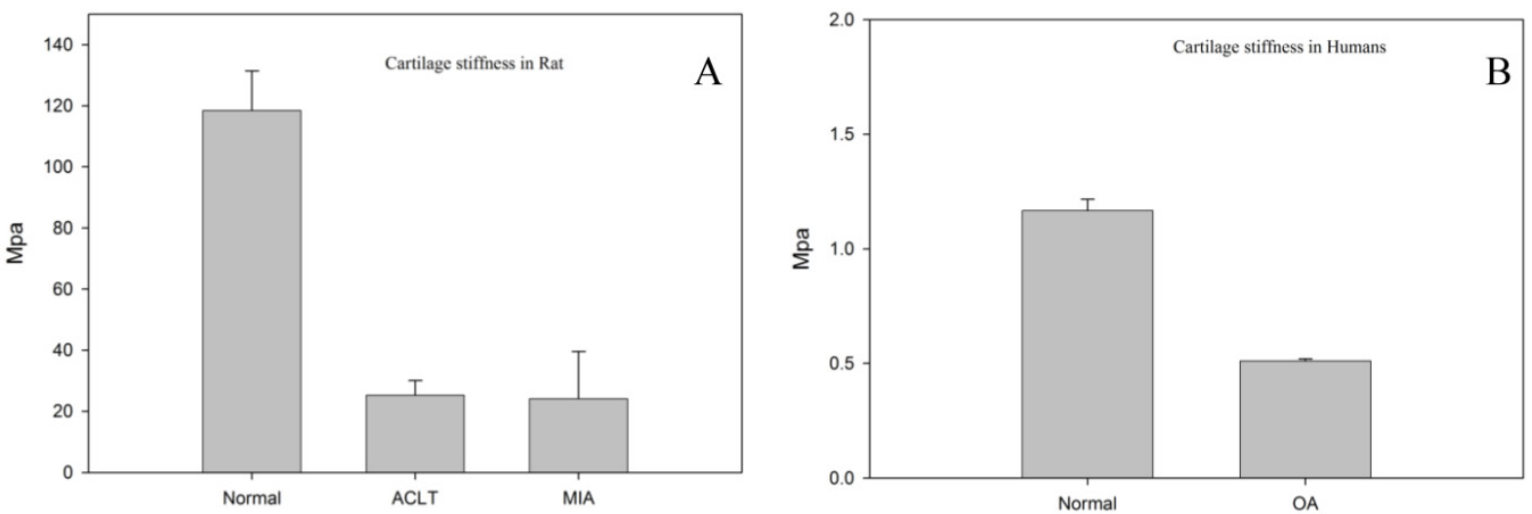

Figure 4: Cartilage stiffness of normal, MIA and ACLT rats (A) and human tissues (B) showed significantly lower cartilage stiffness scores. No significant difference in the cartilage stiffness between MIA and ACLT $(p>0.05)$.

\section{Biomechanical evaluation}

Since our histology results showed more destructive changes at week 2 compared to week 1 (data not shown), the biomechanical evaluation was performed on the samples only at week 2 . Figure $4 \mathrm{~A}$ and $4 \mathrm{~B}$ represents the cartilage stiffness for rat and human, respectively. The intervention groups had significantly lower cartilage stiffness scores (MIA $=24.2 \pm$ $15.3 \mathrm{Mpa}$ and $\mathrm{ACLT}=25.3 \pm 4.8 \mathrm{Mpa}$ ) as compared to that in the normal knees $(118.43 \pm 25.2 \mathrm{MPa})(\mathrm{p}<0.05)$. However, no significant difference was observed between ACLT and MIA group. Analysis of the human tissue samples revealed lower cartilage stiffness score in the human OA group $(0.5 \pm 0.0 \mathrm{MPa})$ as compared to that of the normal cartilage (control group) (1.1 $\pm 0.5 \mathrm{MPa}$; $<0.05)$.

\section{Correlation Analysis}

The results of the correlation analysis between the various parameters (histology, GAG/total protein and biomechanical) assessed in the rat tissue samples are shown in figure 5 . Considering that in week 1 , the GAG/total protein content in the MIA group was significantly higher than in week 2 suggesting that the damage was still progressing, whereas in week 2, the levels were much lower and comparable to that in the ACLT group suggesting that the maximal damaging effect may have been reached in both groups at this time point, hence, only data from week 2 (ACLT and MIA) were included in the correlation analysis to avoid selective biasness. There was a strong correlation between GAG/total protein level and the cartilage stiffness $\left(\mathrm{R}^{2}=0.88,(\mathrm{p}<0.05)\right.$; Fig. $\left.5 \mathrm{~A}\right)$, such that for every 1 unit of GAG/total protein loss, the stiffness reduces by $0.26 \pm 0.03 \mathrm{MPa}$. The GAG/total protein level was also perfectly correlated with the histological score (Mankin) $\left(R^{2}=0.92, p<0.05\right.$; Fig. $\left.5 B\right)$ to the extent that the loss of 1 unit of GAG/TP is associated with $1.2 \pm 0.1$ unit reduction in the Mankin score.
No significant correlation was observed between the cartilage stiffness and histological scores $(\mathrm{p}>0.05)$ (Fig $5 \mathrm{C})$.

\section{DISCUSSION}

The present study demonstrates that chemical induction of osteoarthritis by intra-articular injection of Monosodium Iodoacetate (MIA) in rat model results in cartilage degenerative changes that are comparable to that produced by the anterior cruciate ligament transection method (ACLT) and more importantly to that of human with clinically established OA. This include structural changes such as lack of surface regularities, cartilaginous matrix collapse, cartilage fibrillation as well as functional change such as reduction in the compressive stiffness of the articular cartilage, which will ultimately affect the ability of the tissue to withstand the effect of load-bearing activities.

Previous isolated studies have indicated that intra-articular injection of MIA does not create OA like ACLT or other surgical methods $(16,4)$. Nevertheless, this issue remains debatable and that contrasting reports have also been observed in many literatures (17-19). In this study, we have detected apparent degenerative changes in the articular cartilage of the MIA-induced animals that are comparable to that of ACLT. However, there is a difference in how rapidly the development of OA occurs. It is postulated that this time-dependent progression may be due to the different mode of action in the knee joints (10). Earlier studies have reported that MIA has inhibitory effect on the activity of glyceraldehydes-3 phosphate dehydrogenase in chondrocytes resulting in disruption of glycolysis, hydration of the extracellular matrix, increased extractability as well as reduced quantity and synthesis of proteoglycans, and eventually leads to cell death. Subsequently, the histological and biochemical changes occurred in the articular cartilage of the knee joint bears close resemblance to human OA 
(20-22). The slow chronological progression of OA demonstrated in this study is consistent to that reported by Guzman (23). In contrast, OA in the ACLT model that is the result of mechanical delamination of cartilage causes immediate damage to this tissue, which results in acute inflammatory response. This in turn produces extensive structural changes within a short period $(8,23,24)$. Furthermore, since ACLT is an instability- or biomechanically-induced model, factors such as cage dimensions and flooring could play a role in the development of the disease as they influence the animal's activity on the affected limb (25). In the present study, the GAG/total protein level was significantly lower in ACLT group compared to MIA at week-1 post OA induction but reached similar level 2 weeks after the induction of $\mathrm{OA}$, indicating that ACLT induces destruction of the extracellular matrix of the articular cartilage at a faster rate compared to MIA.
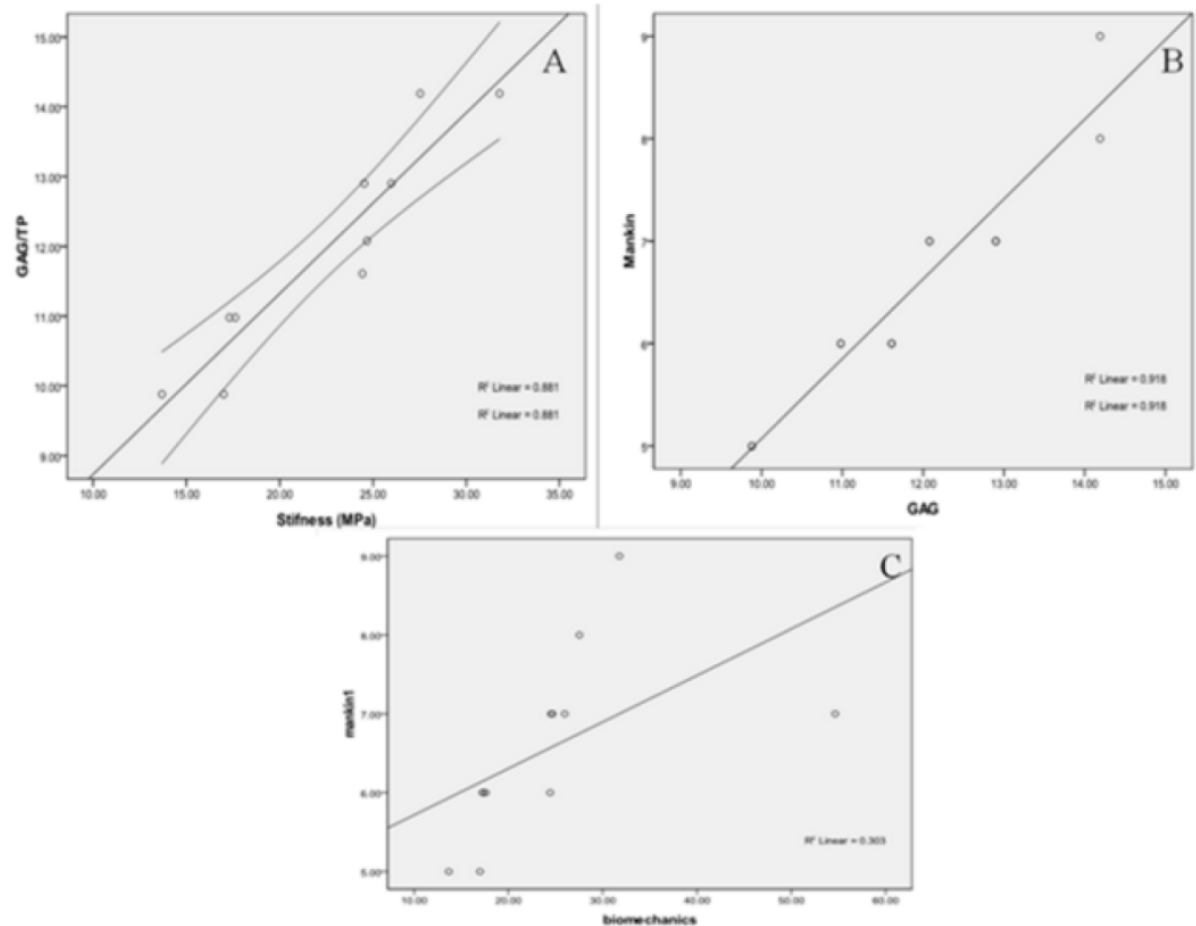

Figure 5: Substantial correlation was observed between GAG/Total protein with two other parameters i.e. cartilage stiffness (A) and histological scores (B). No significant correlation was observed between the histological score and biomechanical testing (C).

It is a well-known fact that, in the highly organized extracellular matrix of articular cartilage, water occupies the intra-molecular and inter-molecular space within a network of collagen fibrils (15\% to $22 \%$ by wet weight) and proteoglycan aggregates (4\% to $7 \%$ by wet weight) $(26,27)$. Studies have demonstrated that along with other factors, proteoglycan content, fixed charge density and molecular organization of the matrix determines the pore size of the tissue, thereby regulating the permeability of the interstitial fluid flow $(28,29)$. The levels of GAG/total protein content were measured in this study to reflect the biochemical changes related to the degenerative process outlined above. Compared to the normal tissue, both ACLT and MIA osteoarthritis rat models showed a marked difference in the altered structure (histopathology) and GAG content 2 weeks post OA induction. Similarly, the human cartilage affected by OA also has markedly reduced GAG/total protein con- tent compared to its healthy counterparts. Higher heterogeneity of the disease in the human tissue samples may explain the observation that the GAG/total protein content in the animal tissues subjected to both OA induction methods remained relatively higher than that in the human OA tissue samples. It was also observed that the differences in the GAG/total protein level were more striking in the femur rather than in the tibia. This may be due to the fact that when a knee undergoes flexion-extension, a series of rolling, sliding and gliding motion is observed between the joint surfaces of the proximal tibia and distal femur (30-33). Because the surface area of the femoral condyle is larger than the tibial plateau, the shearing effect is more likely to be experienced on this surface area, being distributed throughout the articulating surfaces. This results in more articular cartilage being damaged (34-36). Furthermore, the tibial plateau is covered by the meniscus, which will 
have a protective effect against shearing and loading mechanical forces. Thus, the cartilage within the tibial surface is less likely to be affected $(33,37)$. It is also worth noting that after undergoing ACLT transection, the slide and rolling motion that is observed in normal knee kinematics will now be replaced with gliding motions $(38,39)$. This will result in increased articular surface wear and tear on the femoral side as the result of excessive shearing forces being applied. This also leads to increased delamination of the femoral cartilage, hence the increased loss of cartilage from the femoral surface.

To increase the dimension of assessment of the OA changes in the affected tissues, we have incorporated the biomechanical testing to evaluate the tensile strength of the cartilage in response to compression load. Our findings of reduced cartilage stiffness in both animal OA models and human OA appear to be consistent with the findings of others (40). The simultaneous decrease in the GAG content may affect the stiffness and the visco-elastic property of the cartilage. Given that the tensile stiffness and strength of cartilage depend on the organization of the collagen network, denaturing the collagen network subsequently leads to the development of OA (41). Furthermore, recent studies using delayed Gadolinium-Enhanced Magnetic Resonance Imaging of Cartilage (dGEMRIC) have also shown a high correlation between GAG distribution and biomechanical properties of cartilage (42). Similarly, we have also demonstrated a positive correlation between GAG contents with cartilage stiffness in this study. Several studies suggest that a simultaneous determination of the mechanical properties and GAG content of articular cartilage (especially if the GAG content is determined at the site of indentation) may provide valuable information toward understanding the aetiology of OA in articular cartilage, especially during the early stages of the disease process (43-45).

Considering that the degenerative changes induced by MIA is similar to that of ACLT and human OA, together with its additional advantage of being only minimally invasive, reproducible and relatively easy to conduct, MIA can be considered to be a more practical alternative than ACLT as a translational animal model of OA. The onset, progression and severity of OA can be easily controlled in this model by changing the dose of MIA. Guingamp et al. previously showed that the severity of cartilage degradation depends on the dosage of MIA injected into the knee joint (21). A recent preliminary study by Mohan et al indicated that a higher dose of MIA ( $2 \mathrm{mg})$ produced tissue characteristics consistent with severe human OA at 2 weeks post-injection, an observation supported by the present study (17). At a lower dose, the degenerative changes such as loss of tibial articular cartilage, exposure of subchondral bone and osteophytes formation were only apparent at 10 weeks post-injection. MIA model also has an additional advantage of being a suitable model for investigating chronic pain, particularly that associated with OA $(18,19)$. The introduction of MIA to the knee joint results in acute inflammation, leading to the release of pain mediators such as prostaglandins, bradykinin and substance $\mathrm{P}$ in the local area. Although the inflammatory response to the MIA injection has shown to have been largely resolved by 7 days post-injection, it may have a residual effect of sensitising the peripheral receptors, leading to a C-fibre mediated decrease in the threshold of action potential generation and an increase in the afferent nerve transmission to the spinal cord (46). The extended activation of the nociceptive pathways may contribute to the development of chronic pain. Thus, the MIA injection rat OA model can potentially be used to predict the efficacy of anti-nociceptive agents in OA.

Despite the detailed preparation and robust design of this study, several limitations were noted. The main limitation to the present study is in the few numbers of rats used in each group i.e. 18 rats per group and 3 rats at each time point and parameter of evaluation. While this hampers a more convincing conclusion to be drawn from this study, it does provide preliminary data which may justify larger scale studies to be conducted. The use of more objective and sophisticated outcome measures, which may include analysis of cartilage gene expression such as COMP, may have extended the dimension of assessment of the degenerative effect of MIA and ACLT on the animal tissues. Despite being preliminary in nature, we believe that the present study offers valuable data that could promote further use of the MIA injection rat model as translational animal model of OA in future studies.

In conclusion, the findings of this study demonstrate that MIA injection in the rat knee joint effectively induces degenerative changes in the tissues that were comparable to that of the ACLT method and human knee with clinically established OA. In view of its minimal invasiveness and greater convenience of the procedure, increased use of MIA injection as translational animal model of OA should be considered.

\section{ACKNOWLEDGEMENT}

The authors would like to acknowledge High Impact Research - Ministry of Higher Education (HIR-MOHE) and University of Malaya Research Grant (RG 216/10HTM) for their funding. The study sponsors are not involved in the study design; collec- 
tion, analysis and interpretation of data; the writing of the manuscript; the decision to submit the manuscript for publication.

\section{COMPETING INTERESTS}

\author{
The authors have declared that no competing \\ interest exists.
}

\section{REFERENCES}

1. Guccione AA. Arthritis and the process of disablement. Phys Ther. 1994; 74(5): 408-14.

2. Sipe JD. Acute-phase proteins in osteoarthritis. Semin Arthritis Rheu. 1995; 25(2): 75-86

3. Felson DT, Lawrence RC, Hochberg MC, McAlindon T, Dieppe PA, Minor MA, Blair SN, Berman BM, Fries JF, Weinberger M, Lorig KR, Jacobs JJ, Goldberg V. Osteoarthritis: new insights. Part 2: treatment approaches. Ann Intern Med. 2000; 133(9):726-37.

4. Christopher BL and Margaret MS. Animal Models of Osteoarthritis. Curr Rheum Rev. 2008; 4(3): 175-182.

5. Ameye LG, Young MF. Animal models of osteoarthritis: lessons learned while seeking the "Holy Grail". Curr Opin Rheumatol. 2006; 18(5): 537-547.

6. Hayami T, Pickarski M, Zhuo Y, Wesolowski GA, Rodan GA, Duong LT. Characterization of articular cartilage and subchondral bone changes in the rat anterior cruciate ligament transection and mini -sectomized models of osteoarthritis. Bone 2006; 38 (2): 234-243.

7. Setton LA, Elliott DM, Mow VC. Altered mechanics of cartilage with osteoarthritis: human osteoarthritis and an experimental model of joint degeneration. Osteoarthr Cartilage 1999; 7: 2-14.

8. Van der Kraan PM, Vitters EL, van de Putte LB, van den Berg WB. Development of osteoarthritic lesions in mice by "metabolic" and "mechanical" alterations in the knee joints. Am J Pathol. 1989; 135: 1001-14.

9. Fernihough J, Gentry C, Malcangio M, Fox A, Rediske J. Pain related behaviour in two models of osteoarthritis in the rat knee. Pain 2004; 112: 83-93.

10. Bove SE, Calcaterra SL, Brooker RM, Huber CM, Guzman RE, Juneau PL, Schrier DJ, Kilgore KS. Weight bearing as a measure of disease progression and efficacy of anti-inflammatory compounds in a model of monosodium iodoacetate-induced osteoarthritis. Osteoarthr Cartilage 2003; 11: 821-830.

11. Ivanavicius SP, Ball AD, Heapy CG, Westwood FR, Murray F and Read SJ. Structural pathology in a rodent model of osteoarthritis is associated with neuropathic pain: increased expression of ATF-3 and pharmacological characterization. Pain 2007; 128: 272-282.

12. Piscaer TM, Waarsing JH, Kops N, Pavljasevic P, Verhaar JAN, van Osch GJVM and Weinans $H$. In vivo imaging of cartilage degeneration using $\mu \mathrm{CT}$-arthrography. Osteoarthr Cartilage 2008; 16: 1011-1017.

13. Koh YH, Hong SH, Kang HS, Chung CY, Koo KH, Chung HW, Cha JH and Son KR. The effects of bone turnover rate on subchondral trabecular bone structure and cartilage damage in the osteoarthritis rat model. Rheumatol Int. 2009; 30(9): 1165-1171.

14. Mankin HJ, Johnson ME, Lipiello L. Biochemical and metabolic abnormalities in articular cartilage from osteoarthritic human hips. III. Distribution and metabolism of amino sugar containing macromolecules. J Bone Joint Surg. 1981; 63A: 131-9.

15. Landis JR, Koch GG. The measurement of observer agreement for categorical data. Biometrics 1977; 33: 159-174.

16. Smith MM, Little CB. Experimental models of osteoarthritis. In: Moskowitz RW, Altman RD, Hochberg MC, editors. Osteoarthritis. Diagnosis and medical/surgical management. Philadelphia: WB Saunders Company: 2007: 107-25.

17. Geetha Mohan, Egon Perilli, Julia S Kuliwaba, Julia M Humphries, Ian H Parkinson and Nicola L Fazzalari. Application of in vivo micro-computed tomography in the temporal characterisation of subchondral bone architecture in a rat model of low-dose monosodium iodoacetate-induced osteoarthritis. Arthritis Research \& Therapy 2011; 13: R210.

18. Kobayashi K, Imaizumi R, Sumichika H, Tanaka H, Goda M, Fukunari A, Komatsu H. Sodium iodoacetate-induced experimental osteoarthritis and associated pain model in rats. J Vet Med Sci. 2003; 65(11): 1195-9.

19. Combe R, Bramwell S, Field MJ. The monosodium iodoacetate model of osteoarthritis: a model of chronic nociceptive pain in rats? Neurosci Lett. 2004; 370 (2-3): 236-40.

20. Beyreuther, B, Callizot N and Stohr T. Antinociceptive efficacy of lacosamide in the monosodium iodoacetate rat model for osteoarthritis pain. Arthritis Res Ther. 2007; 9: R14-R14

21. Williams VS. Intraarticular hyaluronic acid supplementation in the horse: The role of molecular weight. J Equine Vet Sci. 2007; 27: 298-303.

22. AL-Saffar FJ, Ganabadi S, Yaakub H and Fakurazi S. Collagenase and Sodium Iodoacetate-Induced Experimental Osteoarthritis Model in Sprague Dawley Rats. Asian Journal of Scientific Research 2009; 2: 167-179.

23. Guzman RE, Evans MG, Bove S, Morenko B, Kilgore K. Mono-iodoacetate-induced histologic changes in subchondral bone and artic- ular cartilage of rat femorotibial joints: an animal model of osteoarthritis. Toxicol Pathol. 2003; 31: 619-24.

24. Guingamp C, Gegout-Pottie P, Philippe L, Terlain B, Netter P, Gillet P: Mono-iodoacetate-induced experimental osteoarthritis: a dose-response study of loss of mobility, morphology, and biochemistry. Arthritis Rheum. 1997; 40: 1670-9.

25. Laverty S, Girard CA, Williams JM, Hunziker EB, Pritzker KPH. The OARSI histopathology initiative - recommendations for histological assessments of osteoarthritis in the rabbit. Osteoarthr Cartilage 2010; 18(3): S53-S65.

26. Mow VC, Ratcliffe A. Structure and function of articular cartilage and meniscus. In: Mow VC, Hayes WC, eds. Basic orthopaedic biomechanics. Philadelphia: Lippincott- Raven 1997; 113-177.

27. Muir H. Proteoglycans as organizers of the intercellular matrix. Biochem Soc Trans. 1983; 11: 613- 622.

28. Maroudas A. Physicochemical properties of articular cartilage. In: Freeman MAR, ed. Adult articular cartilage. Kent, England: Pitman Medical Publishing: $1979 ; 215-290$.

29. Mow VC, Holmes MH, Lai WM. Fluid transport and mechanical properties of articular cartilage. J Biomech. 1984; 17: 377-394.

30. Martelli s, Pinskerova V. The shapes of the tibial and femoral articular surfaces in relation to tibiofemoral motion. J Bone Joint Surg (Br) 2002; 84:607-613.

31. Iwaki H, Pinskerova V, Freeman M. Tibiofemoral movement. 1: the shapes and relative movements of the femur and tibia in the unloaded cadaver knee. J Bone Joint Surg (Br) 2000; 82:1189-1195.

32. Johal P, Williams A, Wragg P, Hunt D, Gedroyc W. Tibio-femoral movement in the living knee: an in-vivo study of weight bearing and non-weight bearing knee kinematics, using 'interventional' MRI. J Biomech. 2004; 38(2):269-76.

33. Pinskerova $\mathrm{V}$ et al. The shapes and relative motions of the femur in the unloaded cadever knee. In: Insall JN, scott WN, eds. Surgery of the knee, chap 10, 3rd edn. Philadelphia: Saunders. 2001: 255-283.

34. Hungerford DS, Barry BS. Biomechanics of the patellofemoral joint. Clinical Orthopaedics 1979; 144: 9-15.

35. Wong BL, Kim SH, Antonacci JM, Mcllwraith CW, Sah RL. Cartilage Shear Kinematics During Tibio-Femoral Articulation: Effect of Acute Joint Injury \& Tribosupplementation on Synovial Fluid Lubrication. Osteoarthritis Cartilage. 2010; 18(3): 464-471.

36. Wong BL, Bae WC, Chun J, Gratz KR, Lotz M, Sah RL. Biomechanics of Cartilage Articulation. Effects of Lubrication and Degeneration on Shear Deformation. Arthritis \& Rheumatism, 2008; 58(7): 2065-2074.

37. Helfet AJ. Disorders of the knee 2nd Ed. Philadelphia: Lippincott. 1974:1-37

38. Dargel J, Gotter M, Mader K, Pennig D, Koebke J, Schmidt-Wiethoff R. Biomechanics of the anterior cruciate ligament and implications for surgical reconstruction. Strategies Trauma Limb Reconstr. 2007; 2(1): 1-12.

39. Nirtal Shah. Increasing Knee Range of Motion Using a Unique Sustained Method. N Am J Sports Phys Ther. 2008; 3(2): 110-113.

40. Franz T, Hasler EM, Hagg R, Weiler C, Jakob RP and Mainil-Varlet P. In situ compressive stiffness, biochemical composition, and structural integrity of articular cartilage of the human knee joint. Osteoarthr Cartilage 2001; 9: 582-592.

41. Bae WC, Temple MM, Amiel D, Coutts RD, Niederauer GG, Sah RL. Indentation testing of human cartilage: sensitivity to articular surface degeneration. Arthritis Rheum. 2003; 483: 382-94.

42. Felson DT, Neogi T. Osteoarthritis: is it a disease of cartilage or of bone? Arthritis Rheum. 2004; 50: 341-4.

43. Wang CC, Guo XE, Sun D, Mow VC, Ateshian GA and Hung CT. The functional environment of chondrocytes within cartilage subjected to compressive loading: A theoretical and experimental approach. Biorheology 2002; 39: 11-25.

44. Ericsson YB, Tjornstrand J, Tiderius CJ and Dahlberg LE. Relationship between cartilage glycosaminoglycan content (assessed with dGEMRIC) and OA risk factors in meniscectomized patients. Osteoarthr Cartilage 2009; 17: 565-570.

45. Andriacchi TP, Mundermann A: The role of ambulatory mechanics in the initiation and progression of knee osteoarthritis. Curr Opin Rheumatol. 2006; 18: 514-8.

46. Rang HP, Bevan S and Dray A. Chemical activation of nociceptive peripheral neurones. Br. Med. Bull. 1991; 47: 534-548. 